\title{
Wi-fi based Multi-equipment Universal Remote Controller for Smart Homes
}

\author{
Sumaiya Salim Khan \\ Electronics and Telecommunication Department \\ Dwarkadas J. Sanghvi College of Engineering, \\ Mumbai, India-400056
}

\author{
Vivek Deodeshmukh \\ Electronics and Telecommunication Department \\ Dwarkadas J. Sanghvi College of Engineering, \\ Mumbai, India-400056
}

\begin{abstract}
Presently, we have diversified devices like TV, Set-top box, AC, DVD player etc. which creates confusion of choice of remote controllers (RC) to operate them. Naturally, these multiple RCs requires more number of batteries. With the evolution of electronic equipment's, the range of capabilities or performance has increased but at the same time, the buttons are also increasing on the RC. In addition to this, there is compulsion to have line of sight (LOS) communication in existing devices. To resolve these problems, an Universal Remote Controller (URC) is schemed. This paper focusses at designing Wi-Fi based multidirectional universal RC single unit, that uses Wi-Fi and and internet of things (IOT). This Universal RC is Omni-directional in nature and can handle operations of many devices simultaneously. The prototype used is Air Conditioner (AC). We are able to handle each and every operation of all ACs simultaneously. We are linking the use of URC to IOT. We are creating the mobile phone application (app) that can be a simple chrome like Explorer in which HTML code has been written and buttons are generated through it which handles the functions of all AC's without the compulsion of being in the vicinity of those AC's. Thus there is no need of LOS communication between AC's and RCs. Practically, good communication was observed as $25 \mathrm{~m}$ through walls and $40 \mathrm{~m}$ in open space. This range can be increased by implanting different secondary units in each floor. All the operations can be handled by any smart phone available in the market.
\end{abstract}

\section{General Terms}

Multidirectional, Omni-directional et. al.

\section{Keywords}

IOT, LOS, URC, RCs, smart phone

\section{INTRODUCTION}

Our homes consists of many electronic gadgets like TV,SETTOP BOX, AC, Refrigerator, etc. To make these appliances work, we have RCs for each appliance. Thus, we have different RCs for each of these appliances. RCs have made our lives very easier. RCs have become the basic aspect of our comfort zone. It brings the comfort in our lives. It provokes laziness in humans. Personal comfort level is different from person to person. Every person has its own way of comfortability. RCs have been designed to have the control over these devices. Thus, we can have the user control over these appliances.

RCs are providing us with many options. As the electronics is gaining pace in our lives, the options provided by the RCs are also increasing. Hence, the basic reason to have RC is to make our appliances work as per our desire. Our aim is to design
URC which is Wi-Fi based. We have many electronic devices at our homes. Each of these devices are equipped with their individual RCs. There is a stack of RCs which creates confusion in choosing respective RCs corresponding to their appliance. Each of these RCs will require 2 batteries. Thus WiFi based URC saves batteries because it handles many appliances at a time. For example, if we are operating 4 devices, total number of batteries required are 8 . Thus, WiFi based URC can save up to 6 batteries in that case. URC acts as a single unit which replaces the stack of individual RCs.

This is the world of Internet. There is nothing possible without the use of Internet. IOT is the technology that interconnects the devices and make them feasible to exchange the data or information. IOT connects the devices in smart spaces. It is the ever trending branch as Internet is the basic need of our life. IOT has implemented intelligence in the electronic devices. IOT has been applied to different fields such as Smart Homes [10]. The application fields in Smart Homes incorporate smartness into home areas for comfort, safety, security, health-care and energy conservation [11]. The basic need of humans is the comfort, luxurious and convenient life. Smart Homes provide them with these aspects. Smart Homes is the way of making our electronic equipment's intelligent. For IOT based Smart Homes, Home Automation is the crucial part which needs to be considered. Home Automation systems are used to control home devices or appliances in Smart Homes and provide automatic remote control inside or outside homes [12]. Thus we are linking URC to IOT based Smart Homes. You can handle all the operations of household appliances without being physically present in the home. All the existing devices needs a LOS communication between a device and a RC. WiFi based URC provides NLOS (Non Line Of Sight) communication and thus can be used for home automation. We can have the electronic control of our homes. At the transmitter side, Smart Phone can be used to give the commands to these appliances.

Gang Pan et al. [1] proposed a GeeAir kind of URC into 2010 published Springer journal. They have provided a joystick type of URC which can be used for physically and vision impaired persons. By rotating the GeeAir into different directions, visually impaired user can know which device is in front of them with the help of microphone inbuilt in GeeAir and they can operate the devices through verbal commands. Kuen-Min Lee et al. [2] proposed PnP intelligent URC. The functions related to current device status will only appear on the UI (User Interface).

\section{METHODOLOGY}

Initially, WiFi based URC is a wireless remote which has the feature of IOT. One of the great advantage of this URC is that 
it converts existing LOS remotes by a wireless remote. Fig. 1 is the block diagram of the proposed scheme. At the transmitter side, we have any android or smart phone (SP) on which HTML code is written. This SP will show us the cloned copy of RC. The prototype used is ACs. Our URC will handle the operations of $7 \mathrm{ACs}$ simultaneously in the building. SP will display as " $\mathrm{AC} 1 \mathrm{AC} 2 \mathrm{AC} 3 \mathrm{AC} 4 \mathrm{AC} 5 \mathrm{AC} 6 \mathrm{AC} 7$ ". User will choose one of the $\mathrm{AC}$ onto which operation has to be performed. If user chooses "AC1", the actual clone of the RC of AC1 will appear on the SP. By choosing any desired option onto it, he/she may perform different functions on $\mathrm{AC} 1$. In the building, primary unit is implanted in the LOS of these ACs. Thus, there is multi-directional communication between primary unit and ACs. Now, this primary unit is a hardware unit which has many components. It consists of a AtMega microcontroller. Raspberry-pi3 (R-Pi), Node MCU, TSOP receiver, capacitors, resistors, etc. R-Pi generates its own network and opens the access to SSID passwords. R-Pi is a low cost device that acts as a server and provides the storage of information or data. Secondly, there is Node MCU which acts as a open source IOT platform device which basically communicates with the SP through WiFi created by R-Pi. Node NCU takes the signal from mobile phone and decodes them. These decoded signals are further sent to AtMega 328 controller. For ACs, we need to have temperature feedback and hence there is a need for ADC. AtMega 328 controller has single ADC for temperature feedback.

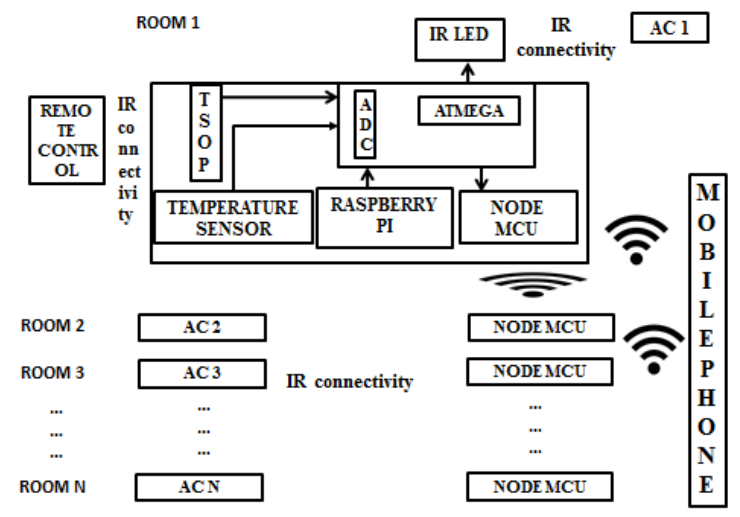

Fig.1. Block Diagram of the Proposed Scheme

\subsection{Working}

The primary unit consists of a power supply section which converts a rechargeable battery of $6 \mathrm{~V} / 1.3 \mathrm{Ahr}$ to $5 \mathrm{~V}$. This conversion is done through 7805 regulator which is a series regulator. Filtering capacitor of $10 \mu \mathrm{F}$ is used to ground all the AC signals and pass only DC signals. WiFi based URC has a auto tuning feature which has the capability to read any random remote signal and whenever in need, stores it corresponding binary digital pattern into R-Pi. In actual, we press the button on the original remote and make the clone or replica of the remote by reading out its digital pattern. This read out of digital signals is done using TSOP receiver. The main feature of this TSOP receiver is that it translates the 38 $\mathrm{KHz}$ signal from our regular remote into binary sequences. These digital sequences are then further stored into R-Pi. Thus the binary sequence is generated for each button. Whenever any button is pressed, the sequence will be generated and the IR on the primary unit will feed it to the instrument. Thus the output of the primary unit will be IR because existing devices still work on IR capability.

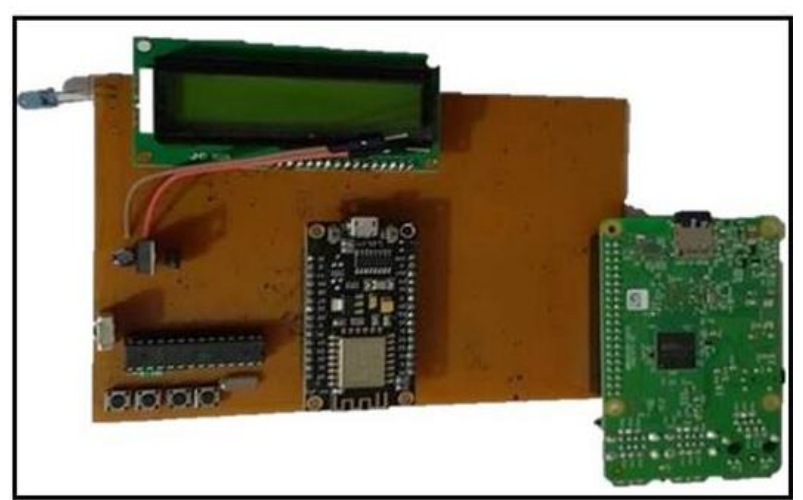

Fig. 2. Hardware Implementation of the Primary Unit

IR module is for all those existing devices which does not have inbuilt WiFi. So in future, if all the devices comes with direct $\mathrm{WiFi}$ module, every home will become smart. There would be no need to create separate WiFi module. As IR cannot penetrate through walls, many secondary units consisting of Node MCU and TSOP can be mounted on each floor to have a good range.

Figure 3 shows the circuit diagram of the primary unit. It consists of a battery of $6 \mathrm{~V}$ which goes to 7805 and modifies it into $5 \mathrm{~V}$. Filtering capacitors are used. Programming in R-Pi is done through python.

TSOP receiver is used to generate binary patterns LCD is used to show the status whether which $\mathrm{AC}$ is working or ACs are $\mathrm{ON} / \mathrm{OFF}$.

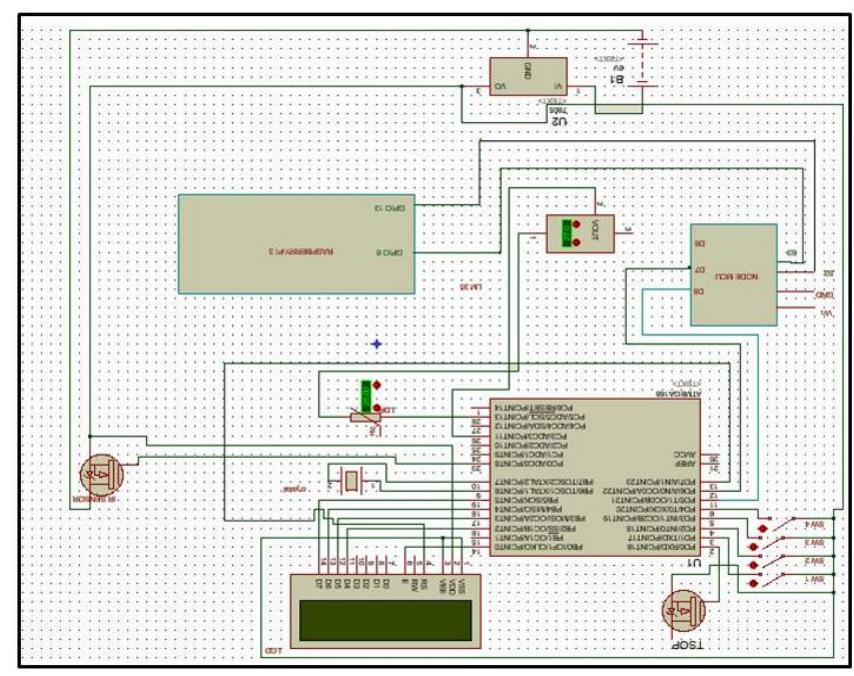

Fig. 3. Circuit Diagram of the Primary Unit

\section{RESULT}

Wi-Fi based URC was tested with 7 ACs simultaneously. Practically, good range was found. There were $3 \mathrm{ACs}$ in one room and rest all ACs were positioned at different places. The tested range was $25 \mathrm{~m}$ through walls and $40 \mathrm{~m}$ in open spaces. This range can even be increased by keeping different secondary units in the building so that whole building can be covered. As it is battery operated, some fluctuations were observed when the battery was draining. Thus it is observed that battery plays important role in getting results.

\section{CONCLUSIONS}

Wi-Fi based URC provides convenience and can be used for 
home automation in Smart Homes. URC has the capability of power saving and equivalent performance with less intricacy. URC can avoid any sort of accidents or fires by giving us the instant feedback on our SP. The prototype used here is ACs. Similarly, TVs, DVD players, etc can also be implemented using this approach. Home Automation can easily be achieved.

\section{ACKNOWLEDGMENTS}

SVKM has given immense support by providing infrastructure and implementing prototypes. We would like to thanks SVKM for the same.

\section{REFERENCES}

[1] Pan, Gang, et al. "GeeAir: a universal multimodal remote control device for home appliances." Personal and Ubiquitous Computing 14.8 (2010): 723-735.

[2] lee et al," point-n-press: an intelligent universal remote control system for home appliances," IEEE transactions on automation science and engineering, vol. 13, no. 3, july 2016

[3] T Kim, et al. "Advanced Universal Remote Controller for Home Automation and Security," IEEE transactions on Consumer electronics , vol. 56, no. 4, November 2010

[4] Masahito Tezuka, et al. "development of bi-directional remote controller protocol and systems for domestic appliances" IEEE transactions on Consumer electronics , vol. 46, no. 3, November 2000.

[5] P. Palanisamy, "A universal device controller (UDC),"
Int. J. Comput.Appl., vol. 82 , no. 10 , pp. 24-29, Nov. 2013.

[6] J. Sathyan and A. R. Ramakrishnan: A Unique SelfContained Universal Remote Control," IEEE Transactions on Consumer Electronics, Vol. 1152 50, No. 4, NOVEMBER 2004",

[7] V. Vujović and M. Maksimović, "Raspberry Pi as a sensor web node for home automation," Comput. Electr. Eng., vol. 44, pp. 153-171, May 2015

[8] K. Gill, S.-H. Yang, F. Yao, and X. Lu, "A ZigBee-based home automation system," IEEE Trans. Consumer Electron., vol. 55, no. 2, pp. 422-430, May 2009.

[9] M. Tezuka, Y. Honda, and M. Kato, "Development of bidirectional remote controller protocol and systems for domestic appliances," IEEE Trans. ConsumerElectron., vol. 46, no. 3, pp. 802-811, Aug. 2000

[10] M. Javier et al., "From the internet of things to the internet of people,"IEEE Int. Comput., vol. 19, no. 2, pp. 40-47, Mar. 2015.

[11] C.-H. Lu et al., "Energy-responsive aggregate context for energy saving in a multi-resident environment," IEEE Trans. Autom. Sci.Eng., vol. 11, no. 3, pp. 715-729, Jul. 2014.

[12] H. C. Tadimeti and M. Pulipati, "Overview of automation systems and home appliances control using PC and microcontroller,” Int. J. Sci. Res., vol.2, no. 4, pp. 127-131, Apr. 2013. 